\title{
Validation of the Chinese version of the Amsterdam Preoperative Anxiety and Information Scale (APAIS)
}

\author{
Hao $\mathrm{Wu}^{\dagger}$, Xin Zhao ${ }^{\dagger}$, Shuaishuai Chu, Fangxia Xu, Jia Song, Zhengliang Ma ${ }^{*+}$ and Xiaoping Gu ${ }^{*+}$
}

\begin{abstract}
Background: Preoperative anxiety is an unpleasant state of tension that may impact patients' post-operative pain and satisfaction. The level of preoperative anxiety should be routinely identified. The Amsterdam Preoperative Anxiety and Information Scale (APAIS) is a self-reported questionnaire that is used to quickly assess preoperative anxiety and information needs with good psychometric properties.
\end{abstract}

Objectives: To validate the Chinese version of the Amsterdam Preoperative Anxiety and Information Scale (APAIS) and to explore coping strategies used by patients in dealing with surgery and anesthetic.

Methods: The cross-cultural validation of APAIS involved the translation of a Chinese version of APAIS and an investigation of its psychometric properties and clinical applicability. Forward-back translation and a pilot study were performed to produce a Chinese adaptation of APAIS. The inpatients of the orthopedic, otolaryngology, and general surgery department scheduled for general anesthesia surgery were enrolled to complete psychometric testing. The reliability was assessed using Cronbach's alpha. Exploratory factor analysis and confirmatory factor analysis were calculated to assess construct validity. The criteria validity was analyzed using the correlation between APAIS and State-trait anxiety inventory-state (STAI-S) and Visual analogue scale-anxiety (VAS-A). Coping styles were evaluated using the Medical Coping Modes Questionnaire (MCMQ) score that covered three domains: confrontation, avoidance, and resignation. The impact of different coping styles on patients' anxiety was explored.

Results: A total of 204 valid questionnaires were collected the day before surgery. Cronbach's alpha coefficients were 0.862 for the anxiety scale and 0.830 for the information scale. Exploratory factor analysis with oblique rotation revealed two factors that explained $76.45 \%$ of the total variances. A confirmatory factor analysis showed a twofactor model with an adequate model fit (root mean square error of approximation: 0.073, goodness-of-fit: 0.966). The APAIS anxiety score significantly correlated with STAI-S $(r=0.717, P<0.01)$ and VAS-A $(r=0.720, P<0.01)$. For the three coping strategies, preoperative anxiety had a low correlation with confrontation $(r=0.33, P<0.01)$ and resignation $(r=0.22, P<0.05)$.

Conclusions: The Chinese version of APAIS is a valid and reliable instrument for assessing preoperative anxiety. Use of this measurement tool for Chinese patients is feasible and shows promising results.

Keywords: Preoperative anxiety, Questionnaires, Validation studies, Coping styles

\footnotetext{
* Correspondence: mazhengliang1964@nju.edu.cn; xiaopinggu@nju.edu.cn

${ }^{\dagger} \mathrm{Hao}$ Wu and Xin Zhao contributed equally to this work and should be considered as co-first authors.

${ }^{\dagger}$ Xiaoping Gu and Zhengliang Ma contributed equally to this work and should be considered as co-corresponding authors.

Department of Anesthesiology, Nanjing Drum Tower Hospital, The Affiliated Hospital of Nanjing University Medical School, 321 Zhong Shan Road,

Nanjing, Jiangsu 210008, People's Republic of China
}

(c) The Author(s). 2020 Open Access This article is distributed under the terms of the Creative Commons Attribution 4.0 International License (http://creativecommons.org/licenses/by/4.0/), which permits unrestricted use, distribution, and reproduction in any medium, provided you give appropriate credit to the original author(s) and the source, provide a link to the Creative Commons license, and indicate if changes were made. The Creative Commons Public Domain Dedication waiver (http://creativecommons.org/publicdomain/zero/1.0/) applies to the data made available in this article, unless otherwise stated. 


\section{Background}

Preoperative anxiety is an unpleasant state of tension and a worldwide problem in the perioperative period. Excessive anxiety can lead to physiological and psychological responses including hypertension, arrhythmias, severer postoperative pain, depression that may impact patients' prognosis and post-operative satisfaction $[1,2]$. As proper psychological intervention can relieve anxiety and improve patient outcomes, patients with a high degree of anxiety should be routinely identified during the anesthesiologist's preoperative interview [2, 3]. Applicable screening instruments that are suitable for preoperative interview settings should be brief and understandable, in addition to reliable and valid [4]. The Amsterdam Preoperative Anxiety and Information Scale (APAIS) is a self-reported questionnaire developed by Moerman in Dutch used to quickly assess preoperative anxiety with good clinical relevance. The scale consists of six items with a simple analysis format. It can be divided into two scales, including an anxiety scale (items 1 , 2, 4, and 5; Cronbach's $\alpha=0.86$ ) and a need-forinformation scale (items 3 and 6; Cronbach's $\alpha=0.72$ ). The APAIS anxiety score is highly correlated with State-trait anxiety inventory-state (STAI-S) [5]. APAIS has been translated into many languages, and the recent version included English, Japanese, French, German, Italian, and Spanish [611]. The cultural difference have been found in evaluation of anxiety. The level of social related anxiety in China was higher than England and Switzer-land, but showed no significant differences compared to Japan and Indonesia. The impact of cultural influences to preoperative anxiety were not clear $[12,13]$. Patient's psychological feeling and satisfaction have been paid more attention in developing countries compared with the past. The high levels of acceptance and clinical relativity make APAIS a standard tool to measure preoperative anxiety that can contribute to improving perioperative psychological feeling in Chinese patients. The primary aim of this study was to develop a Chinese version of APAIS and evaluate its psychometric properties. The correlations between APAIS and STAI-S, Visual analogue scale-anxiety (VAS-A) were evaluated to assess criteria validity. Exploratory factor analysis and confirmatory factor analysis were applied to assess construct validity.

Patients respond to stress in different ways. According to the coping style classified by APAIS, patients who need more information tend to be "blunters", whereas "monitors" require less information support. Coping style is not only an explanatory variability in response to stress, but also an intervention between stress and emotional distress [14]. More specifically, in preoperative stage, "blunter" might benefit from more information and attention. However, extensive information is not useful for those patients with a "blunting" coping style and may even induce excessive anxiety [5]. An important coping task is to regulate stress-induced negative emotions, especially distress [14,
15]. The secondary aim of this research is to explore different coping strategies used by patients in dealing with surgery and anesthetic, and the effect of different coping styles on preoperative anxiety.

\section{Method}

\section{Study population and ethical issues}

This study was approved by the Ethics Committee of the Affiliated Drum Tower Hospital of the Medical Department of Nanjing University (2015-011-01) on March 2, 2015 , and was conducted in accordance with the ethical principles of Helsinki Declaration. All patients were told of their related rights and signed informed consent. Authorization for the Chinese version of APAIS was approved by the original author N. Moerman. Inpatients at the orthopedic, otolaryngologic, and general surgery departments scheduled for general anesthesia surgery between September 2015 were asked to participate in the study. The inclusion criteria were (1) patients undergoing general anesthesia for elective surgery; (2) age > 18 years; (3) physical status of American Society of Anesthesiologists (ASA) I-III; (4) patients were able to read and understand the questionnaire; and (5) they signed informed consent. Patients with psychiatric illnesses, poor medical conditions (heart failure, respiratory failure, shock and multi-organ failure), inability to answer the questionnaire, and who did not consent were excluded.

\section{Development of Chinese version of APAIS}

We translated APAIS according to the rules of the European Organization for the Research and Treatment of Cancer translation procedure [5] and the guidelines of adaptation and validation of instruments in crosscultural health care research [16], which include three main steps: forward translations, blind back-translation, and pilot testing of the preliminary version. A bilingual doctor of anesthesiology and a bilingual graduate majored in English were required to translate APAIS into Chinese. After ambiguities and discrepancies of two translated versions were compared, the initial translated version was generated. Blind back-translation of the initial translated version of were accomplished by two other bilingual independent translators who majored in anesthesiology and English, respectively. The words, sentence structure, semantics of back-translation were discussed and the preliminary version of APAIS was formed. After translation, the preliminary version was applied to the pilot studies; 30 patients (13 males and 17 females) were involved, with an average age of 43 . They completed the questionnaire within 2 minutes and reported no misunderstanding, which ensured that the acceptability for each dimension was good. 
Table 1 List of the 6 APAIS items

\begin{tabular}{ll}
\hline Original items & Chinese items \\
\hline I am worried about the anesthetic. & 我对麻醉感到担心 \\
The anesthetic is on my mind continually. & 我一直在想麻醉这件事 \\
I would like to know as much as possible about the anesthetic. & 我希望尽可能多地了解有关麻醉的事 \\
I am worried about the procedure. & 我对手术感到担心 \\
The procedure is on my mind continually. & 我一直在想手术这件事 \\
I would like to know as much as possible about the procedure. & 我希望尽可能多地了解有关手术的事 \\
\hline
\end{tabular}

\section{Psychometric testing of the Chinese APAIS}

We tested the psychometric properties of the Chinese version APAIS. Data from the APAIS item scores were randomly divided into two groups. Exploratory factor analysis was used in one group to explore the structural relationship between the six items and a confirmatory factor analysis was applied to the other group to verify the structure model. To assess the criteria validity, we analyzed the correlation between APAIS and STAI-S, and VAS-A. In the preoperative stage, STAI-S is a criteria instrument for measuring the current situationrelated anxiety and a widely used instrument that have been translated and validated into Chinese. The VAS-A has characteristics of simple form and well-understood that showed good reliability and validity. The reliability of APAIS anxiety sub-scale and APAIS information sub-

Table 2 Characteristics and APAIS score of the sample

\begin{tabular}{|c|c|c|c|}
\hline & & Total anxiety score & Information desire \\
\hline Variable & n (\%) & Mean (SD) & Mean (SD) \\
\hline \multicolumn{4}{|l|}{ Sex } \\
\hline Male, №. (\%) & 110 & $7.5 \pm 3.3$ & $5.6 \pm 2.7$ \\
\hline Female, №. (\%) & 94 & $9.0 \pm 3.9^{\mathrm{a}}$ & $5.3 \pm 2.6$ \\
\hline \multicolumn{4}{|l|}{ Age } \\
\hline$<50$ & 103 & $8.4 \pm 3.8$ & $5.7 \pm 2.7$ \\
\hline$\geq 50$ & 101 & $8.0 \pm 3.5$ & $5.2 \pm 2.6$ \\
\hline \multicolumn{4}{|l|}{ Department } \\
\hline General surgery & 87 & $8.5 \pm 3.7$ & $5.4 \pm 2.6$ \\
\hline Orthopedic surgery & 82 & $8.0 \pm 3.3$ & $5.7 \pm 2.6$ \\
\hline Otorhinolaryngologic surgery & 35 & $7.9 \pm 4.3$ & $5.1 \pm 2.9$ \\
\hline \multicolumn{4}{|l|}{ History of previous surgery } \\
\hline YES & 90 & $8.1 \pm 4.0$ & $5.0 \pm 2.6$ \\
\hline NO & 114 & $8.3 \pm 3.4$ & $5.8 \pm 2.6$ \\
\hline \multicolumn{4}{|l|}{ ASA Class } \\
\hline ASA I & 103 & $8.0 \pm 3.6$ & $5.5 \pm 2.6$ \\
\hline ASA $\|$ & 76 & $8.4 \pm 3.9$ & $5.1 \pm 2.6$ \\
\hline ASA III & 25 & $8.1 \pm 2.9$ & $6.3 \pm 2.8$ \\
\hline \multicolumn{4}{|l|}{ Surgery } \\
\hline Major & 33 & $9.6 \pm 3.8^{b}$ & $5.2 \pm 2.5$ \\
\hline Medium & 47 & $8.7 \pm 3.5$ & $5.5 \pm 2.8$ \\
\hline Minor & 124 & $7.6 \pm 3.6$ & $6.4 \pm 2.8$ \\
\hline \multicolumn{4}{|l|}{ Education level } \\
\hline Primary Education & 88 & $8.2 \pm 3.8$ & $5.4 \pm 2.8$ \\
\hline Secondary Education & 71 & $8.0 \pm 3.8$ & $5.2 \pm 2.6$ \\
\hline Higher Education & 45 & $8.6 \pm 3.0$ & $5.8 \pm 2.4$ \\
\hline
\end{tabular}

Independent-samples $\mathrm{t}$ test $(\mathrm{t}=2.84, P<0.01)$

${ }^{\mathrm{b}}$ Analysis of variance $(\mathrm{F}=4.14, P<0.05)$ 
scale were assessed using Cronbach's $\alpha$ coefficient. Student's t-test was used to compare the mean scale scores.

\section{Questionnaires \\ APAIS}

APAIS is a 6-item questionnaire that can be subdivided into 2 major components. In the anxiety scale, items 1 and 2 assess anesthesia-related anxiety, and items 4 and 5 evaluate surgery-related anxiety. In the need-forinformation scale, items 3 and 6 assess the desire for information about anesthesia and surgery. All questions range from 1 ("not at all") to 5 ("extremely"). The score of the total anxiety scale ranges from 4 to 20 and 2 to 10 for the information desire score [5].

\section{STAI-S}

STAI-S is a widely used instrument and the gold standard for assessing preoperative anxiety. It was translated and adapted into Chinese with good reliability and validity. It consists of 20 items for measuring the current situation-related anxiety, with scores ranging from 20 to 80 [17].

\section{VAS-A}

The VAS-A consists of a $100 \mathrm{~mm}$ horizontal line; the left edge of the line is marked as "calm," while the other end shows "maximum anxiety." The patients were asked to assess their own anxiety and mark it on the anxiety line [18].

\section{MCMQ}

The coping style was measured using the MCMQ, which is a 20 -item questionnaire that rates on a 4-point Likerttype scale ("never" to "very little;" "all the time" to "very much"). The item scores are summed to produce confrontation, avoidance, and resignation levels, which range from 0 to 27,0 to 21 , and 0 to 15 . The Chinese version of the MCMQ has evidence of satisfactory validity and reliability. In previous study, Cronbach's $\alpha$ of an eight-item confrontation scale, a seven-item avoidance scale, and a five-item acceptance-resignation scale were $0.70,0.66$, and 0.67 , respectively [19].

\section{Data collection}

Two types questionnaires were prepared, the first type of questionnaires containing APAIS, STAI-S, and VAS-A, and the second type of questionnaires containing APAIS, STAI-S, VAS-A and MCMQ. Two kinds of questionnaires with equal number of 120 were randomly to provide to eligible participants the day before surgery. Two hundred twenty-four patients agreed to participate and 204 patients completed the questionnaires before surgery. The distribution and collection of questionnaires were done by one anesthetist. The patients' demographic information was
Table 3 Communalities, factor loadings, eigenvalue, percent of variance in a two-factor model

\begin{tabular}{llllll}
\hline & Communalities & \multicolumn{2}{l}{ Factor Lodings } & Eigenvalue & $\begin{array}{c}\text { Percent } \\
\text { of } \\
\text { variance }\end{array}$ \\
\cline { 3 - 4 } & & F1 & F2 & & $46.53 \%$ \\
\hline A1 & 0.60 & -0.70 & 0.33 & 3.59 & \\
A2 & 0.69 & -0.78 & 0.28 & & \\
A4 & 0.84 & -0.89 & 0.19 & & \\
A5 & 0.79 & -0.87 & 0.17 & & \\
A3 & 0.88 & 0.20 & -0.92 & 1.00 & $29.92 \%$ \\
A6 & 0.79 & 0.29 & -0.84 & & \\
\hline
\end{tabular}

collected, included age, sex, educational background, previous surgeries, and underlying illnesses. The surgery type was divided into minor, intermediate, and major surgery as in the original version.

\section{Results}

\section{Study population and item scores}

The Chinese version of APAIS is shown in Table 1. A total of 204 valid questionnaires were collected the day before surgery; 101 respondents completed the anxiety MCMQ and there were no significant differences between the demographic data of two types questionnaires. The participants were $53.9 \%$ male and $46.1 \%$ female, and the mean age of the inpatients was $47.4 \pm 14.8$ years. Overall, $44.1 \%$ of the participants had a history of previous surgeries. The respondents' demographic characteristics and mean APAIS scores are shown in Table 2. The mean of total anxiety score was $8.2 \pm 3.6$, the anesthesiarelated anxiety score was $3.7 \pm 1.8$, the surgery-related anxiety score was $4.5 \pm 2.2$, and the information desire component was $5.4 \pm 2.6$.

The female participants had significantly higher anxiety scores than the males $(7.54 \pm 3.27$ vs $8.97 \pm 3.93 ; P<$ 0.01 ). The patients undergoing major surgery had higher anxiety scores than those having medium-minor operations $(\mathrm{F}=4.14, P<0.05)$. There were no differences observed in the ratings of the APAIS anxiety score regarding age, department, ASA class, prior surgical procedures, or educational level. Patients with APAIS information sub-scale score $\geq 8$, (score ranges: $2-4,5-7$, and $8-10)$ had a higher anxiety score $(\mathrm{F}=17.86, P<0.01)$.

\section{Reliability and validity of Chinese APAIS}

The internal consistency of APAIS assessed using Cronbach's alpha was 0.862 for the anxiety sub-scale and 0.830 for the information sub-scale. The construct validity was

Table 4 Index of model fitting of a Two-Factor model

\begin{tabular}{llllllllll}
\hline df & $X^{2}$ & $X^{2} / \mathrm{df}$ & $P$ Value & NFI & CFI & GFI & RFI & RMR & RMSEA \\
\hline 7 & 10.86 & 1.551 & 0.145 & 0.965 & 0.987 & 0.966 & 0.925 & 0.049 & 0.073 \\
\hline
\end{tabular}


investigated using factor analysis. An exploratory factor analysis (EFA) with an oblique rotation of 99 subjects revealed two factors, which explained $76.45 \%$ of the total variances: anxiety (items 1, 2, 4 and 5) and the need for information (items 3 and 6). Communalities, factor loadings, eigenvalue, and the percent of variance in a two-factor solution are shown in Table 3. A confirmatory factor analysis (CFA) of 105 participants showed a two-factor model with an adequate model fit, and Goodness of fit index (GFI) and Root mean square error of approximation index (RMSEA) were 0.966 and 0.073 , respectively. The indexes of model fitting are shown in Table 4 . The criteria validity was evaluated by measuring the correlation between the APAIS anxiety score and the STAI-S scores. The mean scores of STAI-S and VAS-A were $38.7 \pm 11.2$ and $3.2 \pm$ 2.5 , respectively. APAIS strongly correlated with STAI-S $(r=0.717, P<0.01)$ and VAS-A $(r=0.720, P<0.01)$.

\section{Coping style and preoperative anxiety}

For the 101 inpatients who completed the MCMQ, the mean values of confrontation, avoidance, and resignation were $18.8 \pm 3.4,15.5 \pm 3.2$, and $7.5 \pm 2.4$, respectively. The correlations between the APAIS anxiety score, information desire score, and the MCMQ are shown in Table 5. At the APAIS anxiety sub-scale cut-off score of 11, 33.7\% of the patients were classified as anxious. For the anxious inpatients, the t-test revealed the differences of each of the three factors: confrontation ( $\mathrm{t}=2.45, P=0.016)$, avoidance $(\mathrm{t}=0.32, P=0.751)$, resignation $(\mathrm{t}=1.38, P=0.169)$, and the information desire score $(\mathrm{t}=2.73, P=0.007)$.

\section{Discussion}

The psychometric evaluation of the Chinese version of APAIS met the expected criteria. The scales showed a high reliability for both anxiety sub-scale and information sub-scale with Cronbach's alpha 0.862 and 0.830 respectively. The criteria validity of Chinese version of APAIS was measured by the correlation with STAI-S, which is considered as gold standard for assessing state anxiety and the calibration of original version of APAIS, showed good efficiency [5, 17]. As in the original study two factors were extracted by EFA, the same results were also observed in our research. The Chinese APAIS differs from the French version with a three-factor structure and the Spanish version with one factor analyzed by CFA $[9,11]$. We further verified the two-factor model for Chinese participants, and the results showed an adequate model fit. The CFA two-factor model coincided with EFA outcomes, which ensured stable construct validity for the new version of APAIS.

Unspecific anxiety questionnaires such as STAI-S were impractical and time-consuming. In our study, APAIS could be completed in 2 minutes with a high response rate and good acceptability in Chinese patients. In pilot testing, 30 participants were asked to rate the items of the scale using a dichotomous scale (clear or unclear) All patients reported clear and no misunderstanding. The possible reasons might be that, first the response format of APAIS was simple, sentence patterns of the six items were similar and colloquial. Second, as a condition-specific scale, APAIS describing surgery and anesthesia was pertinent and accessible in preoperative stage. Third, in the processes of translation/back translation, we solve most issues about words, sentence structure, semantics.

The APAIS contains two subscales: the anxiety scale has already been sufficiently studied, whereas there was relatively little research on the information scale that detects patients coping styles $[8,9]$. Coping, defined as the cognition and behaviors to manage the internal and external demands in stressful situations, was a relatively stable characteristic [20, 21]. In our research, the mean information desire sub-scale score was $5.4 \pm 2.6$, lower than the sample in developed countries, which means more patients choose "blunting" coping style in dealing with surgery and anesthesia in China. It is possible that coping styles and subjective emotions are different across cultures [22]. However, patients with high information requirements or confrontation coping style still had higher anxiety levels [7, 9]. The explanation could be confrontation tended to focus on health-related threatening situations and paying close attention to details, which was an anxiety-provoking way to deal with uncontrollable stressors [23]. Furthermore, the monitoring style was an independent predictor of postoperative pain [24]. Avoidance and resignation were two additional types of coping strategy. Patients with resignation were focused on feelings of impotence and defeat [25]. Avoidance coping tended to avert stressful situations and avoid medical information. The relativities between these two coping styles and preoperative anxiety or information desire were not found in the study.

In addition, we found that the patients undergoing major surgery were more anxious, but the sample size of major surgery was small. Further qualified research is needed to confirm this result. One limitation of this study was that the research sample only included patients undergoing

Table 5 The Pearson correlations of total anxiety, information desire and the coping styles

\begin{tabular}{lllll}
\hline & Confrontation & Avoidance & Resignation & Information desire \\
\hline Total anxiety & $r=0.33, P<0.01$ & $r=0.07, P>0.05$ & $r=0.22, P<0.05$ & $r=0.40, p>0.05$ \\
Information desire & $r=0.23, P<0.05$ & $r=-0.10, P>0.05$ & $r=0.01, P>0.05$ & 1 \\
\hline
\end{tabular}


orthopedic, otolaryngologic, and general procedures. Although the range of surgery types was relatively narrow, in the original APAIS and other adaptions, there are was no interdependency between the anxiety levels and the types of procedures. The inpatients of the three departments were considered adequate to meet research needs.

\section{Conclusion}

The Chinese version of APAIS is a valid and reliable instrument for assessing preoperative anxiety in preoperative interviews. Using this instrument in Chinese patients is feasible and shows promising results.

\section{Abbreviations}

APAIS: The Amsterdam Preoperative Anxiety and Information Scale; CFI: Comparative fit index; Cl: Confidence interval; EFA: Exploratory factor analysis; GFI: Goodness of fit index; ICC: Intraclass correlation coefficient; NFI: Normed fit index; RMSEA: Root mean square error of approximation index; STAI-S: State-trait anxiety inventory-state; VAS-A: Visual analogue scaleanxiety

\section{Acknowledgements}

The authors acknowledge Dr. Yue Liu, Ph.D. and Dr. Tianjiao Xia, Ph.D. for doing translation efforts, Yanjun Liu, M.D. for providing quality checks on translations. and we would thank to the nurses who collaborated in the data collection.

\section{Authors' contributions}

$\mathrm{HW}$ and $\mathrm{XZ}$ participated in study design, data collection and analyze the data. ZM and XG were leading the translation procedures and reviewed the successive versions, and participated in study design. HW, SC and FX analyzed the data and helped with study design. HW and XZ wrote major parts of the paper; XG and JS assisted with writing and editing. All authors read and approved the final manuscript.

\section{Funding}

This research was supported by National Natural Science Foundation of China $(81371207,81171047)$, Natural Science Foundation of Jiangsu Province of China (BK20170129), and Key Talent's of 13th Five-Year Plan for Strengthening Health of Jiangsu Province (ZDRCA2016069).

\section{Availability of data and materials}

The dataset used during the current study is available from the corresponding author on reasonable request.

\section{Ethics approval and consent to participate}

This study was approved by the Ethics Committee of the Affiliated Drum Tower Hospital of the Medical Department of Nanjing University (2015-01101) on March 2, 2015, and was conducted in accordance with the ethical principles in the Declaration of Helsinki. Written consent was obtained from all the participants in the study.

\section{Consent for publication}

Manuscript does not contain any personally identifiable information on research participants.

\section{Competing interests}

The authors declare that they have no competing interests.

Received: 2 January 2018 Accepted: 12 February 2020

Published online: 11 March 2020

\section{References}

1. Norris W, Baird WLM. Pre-operative anxiety - a study of incidence and aetiology. Br J Anaesth. 1967;39(6):503-9.

2. Kiecolt-Glaser JK, Page GG, Marucha PT, et al. Psychological influences on surgical recovery - perspectives from psychoneuroimmunology. Am Psychol. 1998:53(11):1209-18.
3. Kalkman JC, Visser K, Moen J, Bonsel JG, et al. Preoperative prediction of severe postoperative pain. Pain. 2003;105(3):415-23.

4. Laufenberg-Feldmann R, Kappis B. Assessing preoperative anxiety using a questionnaire and clinical rating: a prospective observational study. Eur J Anaesthesiol. 2013;30(12):758-63.

5. Moerman N, van Dam FS, Muller MJ, et al. The Amsterdam preoperative anxiety and information scale (APAIS). Anesth Analg. 1996;82(3):445-51.

6. Boker A, Brownell L, Donen N. The Amsterdam preoperative anxiety and information scale provides a simple and reliable measure of preoperative anxiety. Can J Anaesth. 2002;49(8):792-8.

7. Nishimori M, Moerman N, Fukuhara S, et al. Translation and validation of the Amsterdam preoperative anxiety and information scale (APAIS) for use in Japan. Qual Life Res. 2002;11(4):361-4.

8. Berth H, Petrowski K, Balck F. The Amsterdam preoperative anxiety and information scale (APAIS) - the first trial of a German version. Psychosoc Med. 2007:4:1-8.

9. Axel MS, Loundou A, Capdevila X, et al. Validation of the French version of the Amsterdam preoperative anxiety and information scale (APAIS). Health Qual Life Outcomes. 2013;11(166):1-7.

10. Buonanno $P$, Laiola A, Palumbo $C$, et al. Italian validation of the Amsterdam preoperative anxiety and information scale. Minerva Anestesiol. 2017;83(7): 705-11.

11. Vergara-Romero M, Morales-Asencio JM, Morales-Fernández A, et al. Validation of the Spanish version of the Amsterdam preoperative anxiety and information scale (APAIS). Health Qual Life Outcomes. 2017;15(1):120.

12. Stein DJ. Social anxiety disorder in the west and in the east. Ann Clin Psychiatry. 2009;21(2):109-17.

13. Kan W, Lingyu L, Tomoko $K$, et al. Reliability and validity of the Chinese version of the TaijinKyofusho (TKS) scale. Chin J Clin Psychol. 2018;26(1):35-8.

14. Montero-Marin J, Prado-Abril J, Piva Demarzo MM, Gascon S, GarciaCampayo J. Coping with stress and types of burnout: explanatory power of different coping strategies. PLoS One. 2014;9(2):e89090.

15. Folkman S. Positive psychological states and coping with severe stress. Soc Sci Med. 1997;45(8):1207-21.

16. Sousa VD, Rojjanasrirat W. Translation, adaptation and validation of instruments or scales for use in cross-cultural health care research: a clear and user-friendly guideline. J Eval Clin Pract. 2011;17(2):268-74.

17. Spielberger CD, Gorsuch RL, Lushene RE. State trait anxiety inventory manual. Palo Alto: Consulting Psychologists Press; 1970.

18. Abend R, Dan O, Maoz K, et al. Reliability, validity and sensitivity of a computerized visual analog scale measuring state anxiety. J Behav Ther Exp Psychiatry. 2014;45(4):447-53.

19. Xiaohong S, Qianjin J. Report on application of Chinese version of MCMQ in 701 patients. Chin J Behav Med Sci. 2000;9(1):18-20.

20. Ray C, Lindop J, Gibson S. The concept of coping. Psychol Med. 1982;12(2): 385-95.

21. Roussi $\mathrm{P}$, Miller SM. Monitoring style of coping with cancer related threats: a review of the literature. J Behav Med. 2014:37(5):931-54.

22. Sand M, Hessam S, Sand D, et al. Stress-coping styles of 459 emergency care physicians in Germany: a pilot study. Anaesthesist. 2016;65(11):841-6.

23. Aust $H$, Rüsch $D$, Schuster $M$, et al. Coping strategies in anxious surgical patients. BMC Health Serv Res. 2016;12(16):250.

24. Miller SM, Mangan CE. Monitoring-blunting cognitive coping style, preoperative information and preoperative anxiety. A systematic review. J Pers Soc Psychol. 1983;45(1):223-36.

25. Büssing A, Matthiessen PF, Mundle G. Emotional and rational disease acceptance in patients with depression and alcohol addiction. Health Qual Life Outcomes. 2008;21(6):4.

\section{Publisher's Note}

Springer Nature remains neutral with regard to jurisdictional claims in published maps and institutional affiliations. 